\title{
AN UNUSUAL CARDIAC ANEURYSM IN A YOUNG ADULT
}

\author{
BY \\ J. HIGGINSON AND K. J. KEELEY \\ From the South African Institute for Medical Research, Johannesburg, and Baragwanath \\ Non-European Hospital
}

(RECEIVED FOR PUBLICATION FEBRUARY 22, 1951)

It is generally agreed that the majority of cardiac aneurysms occur in elderly individuals following coronary occlusion. Aneurysms of the heart due to other causes are very rare and are seen with equal frequency at all ages. Most of these occur in the left ventricle, and, for all practical purposes, an origin from other parts of the heart can be neglected.

In the excellent review of the subject by Parkinson, Bedford, and Thomson (1938), the youngest case described was that of a woman of 42 years, who had an aneurysm at the apex of the heart. Aschoff nodules and an acute necrotizing arteritis with focal myofibrosis were found at necropsy. Burn, Hollander, and Crawford (1943) discussed the case of a girl of 13 years who had an aneurysm of the left ventricle in the region of the mitral ring. Although the lesions of active rheumatic fever were present, the authors considered a developmental weakness of the mitral valve was the most probable cause. Vivas-Salas (1948) demonstrated radiologically a ventricular aneurysm in a boy of 7 years which he believed was congenital in origin. An aneurysm of the ventricle in a man of 32 years which ruptured and caused death is described by Berlin and Hallen (1938). No evidence of coronary infarction was found, but the aneurysm appeared to arise in an area of myofibrosis, the aetiology of which was obscure. Three native boys of 6,7 , and 12 years respectively who had ventricular aneurysms are described by Macfie and Ingram (1920). The myocardium showed patchy fibrosis, but no specific aetiological factor was noted, although syphilis was considered a possibility in one case. Diverticula of the heart (Abbott, 1927 ; Roessler, 1944), which are usually found in infants, are sometimes described as aneurysms, but are only of academic interest. The other forms of cardiac aneurysms which have been described in the younger age groups are usually associated with anomalies of the coronary arteries (Bland, White, and Garland, 1933 ; Sanes and Kenny, 1934). We feel, therefore, that the following case of an aneurysm in a young adult is of interest, as the clinical and pathological findings were unusual, and we have been unable to discover a similar case in the English literature. Unfortunately Sternberg's (1914) monograph is not available in this country.

\section{Clinical History}

The patient, an unmarried Bantu woman aged 19 years, was admitted on May 10, 1950, to Baragwanath Non-European Hospital, complaining of a " throbbing " pain to the left of the sternum. A history of an operation for an abdominal swelling several 
years previously was given, but no further information as to the exact nature of the lesion could be obtained. However, from details furnished by the patient, it was believed to have been an ovarian cyst. There was no history of rheumatic fever.

On examination a diastolic murmur was heard at the mitral area, but examination of the rest of the cardiorascular system showed no abnormality. As she had a raised temperature $\left(99^{\circ} \mathrm{F}\right.$ ), tachycardia, and an erythrocyte sedimentation rate of $30 \mathrm{~mm}$. per hour (Westergren), a diagnosis of mitral stenosis and active rheumatic fever was made. The only abnormality in the chest seen radiologically (Fig. 1) was elevation of the left main bronchus which was interpreted as due to a dilatation of the left atrium. The serological tests for syphilis were negative.

Her condition improved on bed rest, and the temperature and E.S.R. fell, but a slight cough developed. She died unexpectedly 15 days after admission and death was considered due to acute cardiac failure.

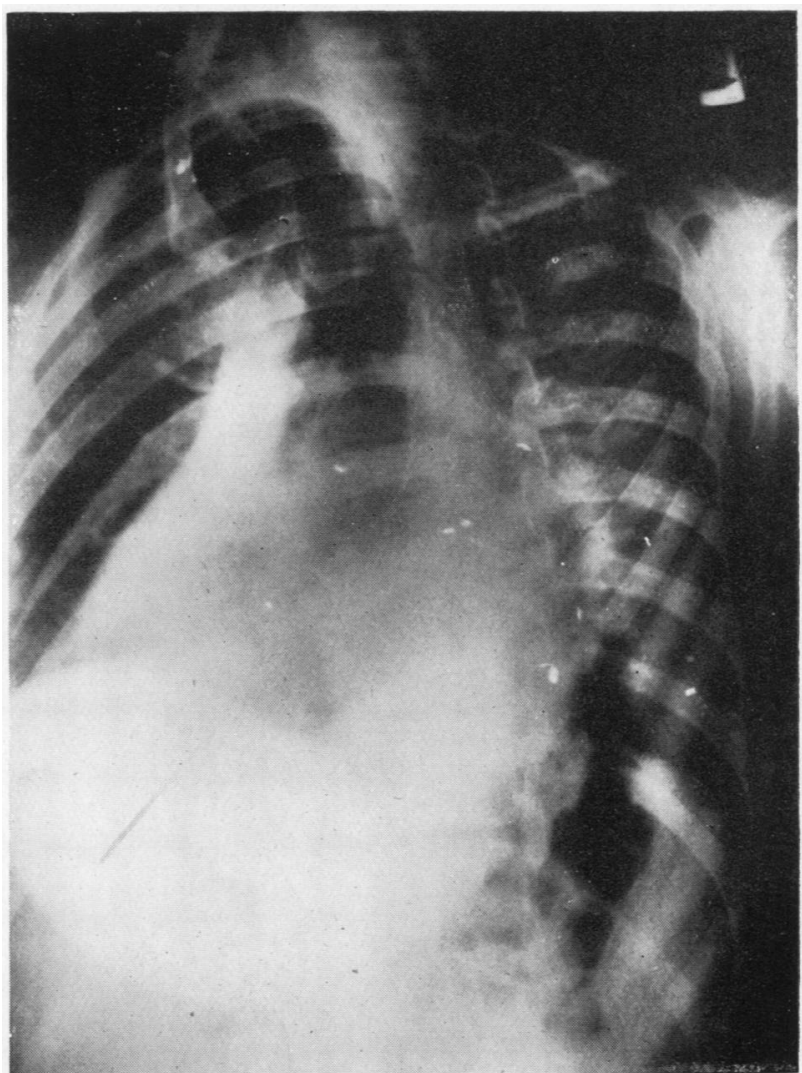

FIG. 1.-Skiagram of the chest in left anterior oblique position. The elevation of the left main bronchus is visible, also the posterior bulging of the heart which was considered a dilated left atrium.

\section{Post-mortem Findings}

The body was that of a young Bantu female with a healed surgical midline incision in the lower abdomen. The fingers were not clubbed and only slight sacral oedema was observed.

The lungs (R. 500 g., L. 300 g.) showed moderate congestion and oedema, especially of the right lower lobe. On section there was congestion of the walls of the alveoli, some of which contained red blood cells and oedema fluid with a few haemosiderin-laden macrophages.

The hilar lymph nodes and spleen showed nodules varying from 1-4 $\mathrm{mm}$. in diameter, which on section were confirmed as caseous tubercles.

The liver $(1,400 \mathrm{~g}$.) was dark red and blood oozed from the cut surface. On section the central veins and adjacent sinusoids were seen to be dilated, with compression and atrophy of the surrounding parenchymal cells. The histological features were those of subacute passive venous congestion.

The spleen $(100 \mathrm{~g}$.) was dark red and firm. The cut surface showed a few scattered necrotic nodules $1-5 \mathrm{~mm}$. in diameter, which, on microscopic examination, showed the typical structure of caseous tubercles. In the surrounding splenic pulp the sinusoids were prominent and congested, but no evidence of increased fibrosis or chronic passive congestion was observed. 
The kidneys (each $75 \mathrm{~g}$.) showed scattered areas of cortical scarring, apart from which the cortex and medulla appeared normal with well-preserved vascular markings. No gross lesions were noted in the renal pelves or calyces. The cortex contained linear areas of interstitial fibrosis in which some of the glomeruli were partially sclerosed, with pericapsular fibrosis and atrophy of the corresponding tubules. Diffuse infiltration of these areas by lymphocytes and plasma cells was present. Some of the medium and small arteries showed a moderate degree of fibrous hyperplasia of the intima, but the majority were healthy. Slight perivascular lymphocytic infiltration and dilatation of the sub-epithelial capillaries of the pelves were noted, but, apart from passive congestion, the remaining cortex and medulla were healthy. The features were those of healed chronic pyelonephritis.

The left ovary and Fallopian tube were absent. It was considered that they had been removed surgically at the former operation.

Except for moderate congestion no pathological lesions were observed in the remainder of the gastro-intestinal and genito-urinary tracts. No other vascular lesions were found apart from those described in the heart.

The heart weighed $500 \mathrm{~g}$. The pericardial sac was completely obliterated by fine fibrous adhesions. On examination a thin-walled sac $(5 \mathrm{~cm}$. in diameter) was observed arising from the region of the mitral ring on the postero-lateral surface (Figs. 2 and 3). In the upper portion of the sac there was laminated thrombus, the remainder containing blood. It communicated with the left ventricle by an opening $(1 \mathrm{~cm}$. in diameter) which was lateral to the mitral valve and unguarded by cusps. The aneurysm was superficial except for a common septum with the left atrium. The septum extended from the base of the heart to the mitral valve and was lined on the atrial side by endocardium and on the aneurysmal side by a thin layer of fibrin. Otherwise the atria were normal, with healthy appendices, and the foramen ovale was closed. The mitral valve was only $1.5 \mathrm{~cm}$. in diameter, but the cusps and chordae tendineae were healthy. At the lateral commissure the cusps were continuous with the septum between atrium and aneurysm.

On the inferior surface the myocardium of the left ventricle merged into a band of fibrous tissue $(3 \mathrm{~mm}$. thick) which formed a gutter between the ventricle and the inferior cusp of the mitral valve (Fig. 4) and merged into the wall of the aneurysm.

The left ventricle showed only a slight dilatation, but the right was hypertrophied, the wall being $7 \mathrm{~mm}$. thick. No abnormality was found in the aortic, pulmonary, and tricuspid valves.

Examination of the coronary arteries showed right coronary preponderance, the right artery supplying the posterior interventricular branch and the base of the heart as far as the left border. On macroscopic examination no lesions were observed in this vessel.

The first part of the left coronary artery and anterior interventricular branch were healthy. The circumflex branch, which was very small, narrowed $3 \mathrm{~cm}$. from its origin and could only be traced with difficulty between aneurysm and ventricle to the left border of the heart, where it ceased. 

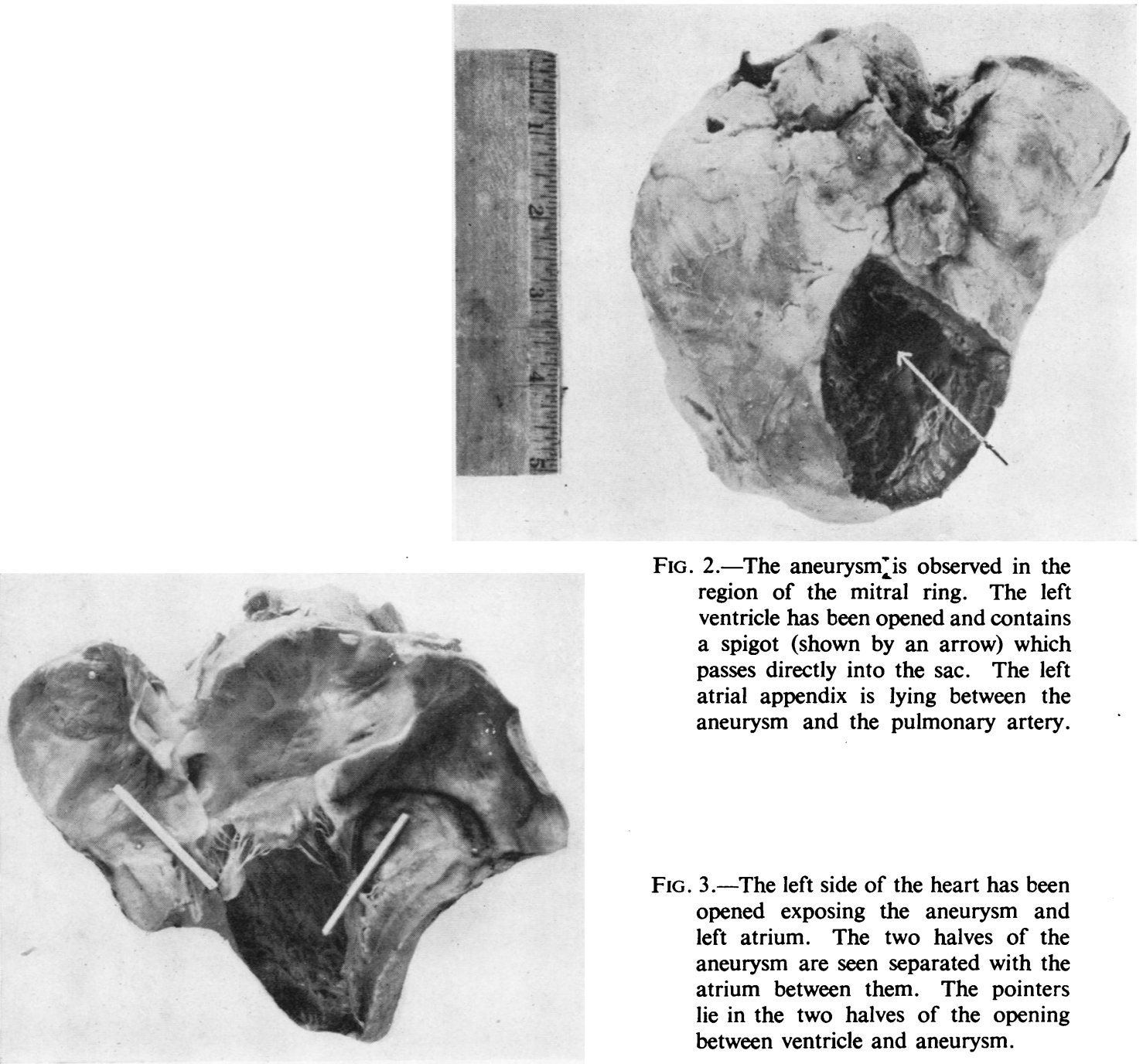

FIG. 2.-The aneurysm is observed in the region of the mitral ring. The left ventricle has been opened and contains a spigot (shown by an arrow) which passes directly into the sac. The left atrial appendix is lying between the aneurysm and the pulmonary artery.

No abnormality was present in the aorta and great vessels.

Microscopical Examination. - The wall of the aneurysm was composed of relatively acellular collagenous tissue. There were a few bands of cardiac muscle near the mitral ring, but they did not extend into the sac, whose wall was almost avascular apart from a few dilated capillaries on the epicardial surface. An endothelium was absent, and the sac was lined by a thin deposit of fibrin. The septum between atrium and aneurysm showed the same histological features, but the rest of the left atrial wall was normal. In the groove between the aneurysm and left ventricle the circumflex artery showed concentric fibrous hyperplasia of the intima with considerable diminution of the lumen. The internal elastic lamina was intact, but a few newly formed elastic fibres were observed in the thickened intima. The media was only slightly fibrosed. The left cardiac vein showed thickening and fibrosis of media and intima on the side adjoining the artery, but the remainder of the vessel wall was normal. The concentric hyperplasia of the intima did not suggest organization of a previous thrombus, and no evidence of active inflamma- 
tory changes was found in the vessel wall or surrounding tissue. The histological features in the artery suggested a reactive endarteritis to some former irritant. In addition many of the small branches of the circumflex artery showed an obliterative endarteritis with almost complete obstruction. These changes in the small vessels extended to the inferior interventricular sulcus in the region of the fibrous gutter supplied by the right coronary artery. The major branches of the right and left coronaries elsewhere showed only patches of very slight intimal fibrous hyperplasia (Fig. 4).

The myocardium of the left ventricle was infiltrated by lymphocytes, mononuclear cells, and a few eosinophils extending diffusely between the muscle fibres (Fig. 5) ; an occasional focus was found with a perivascular distribution. No tubercle bacilli, spirochaetes, or other parasites were found by appropriate staining methods, but unfortunately culture was not performed at the time of the necropsy. In addition several foci of interstitial fibrosis associated with destruction of the muscle fibres were found, the largest being $2 \times 2 \mathrm{~mm}$. in diameter.

The gutter between the ventricle and the inferior mitral cusp was composed of hyaline collagenous tissue lined by a thin layer of fibrin. There were a few scattered bands of muscle fibres on the external surface.

The epicardial tissues were slightly infiltrated by scanty mononuclear cells and eosinophils, and the pericardial space was replaced by loose connective tissue.

In none of the many sections of the heart were Aschoff nodules found and no pathological lesions were observed in endocardium or valves.

The cause of death was attributed to acute right-sided heart failure due to obstruction of the mitral valve by a cardiac aneurysm.

\section{Discussion}

The narrowing of the mitral valve explained the presence of the murmur during life, the pyrexia being caused by either the myocardial or tubercular lesions. However, the pathogenesis of the aneurysm was more uncertain.

The following pathological conditions are listed by Parkinson, Bedford, and Thomson (1938) as the commoner causes of cardiac aneurysms.

Arteriosclerosis of the coronary vessels causes myomalacia and later fibrosis of the myocardium with resultant aneurysm formation. This is by far the commonest cause. Ten cases are described by Dressler and Pfeiffer (1940); 11 cases by Crawford (1943) ; 13 cases by Parkinson, Bedford, and Thomson (1938), but none of these occurred in the younger age groups.

An aneurysm secondary to a syphilitic gumma is rare, but cases are described (Sohval, 1935). One of us saw a multiloculated aneurysm in a male native of 39 years which was almost certainly of this nature.

Mycotic aneurysms are not uncommon, and may involve valve or myocardium. They follow infective endocarditis and are usually by themselves of little additional significance, but a case is described by Benson, Hunter, and Manlove (1933) in which rupture of such an aneurysm led to death.

Rheumatic aneurysms are believed to result from rheumatic necrosis, but are uncommon. Crawford (1943) states that " necrosis of the myocardium secondary to rheumatic lesions has been stated to be an aetiological factor but must be very rare." However, two of the cases mentioned in the introduction appear to be of 


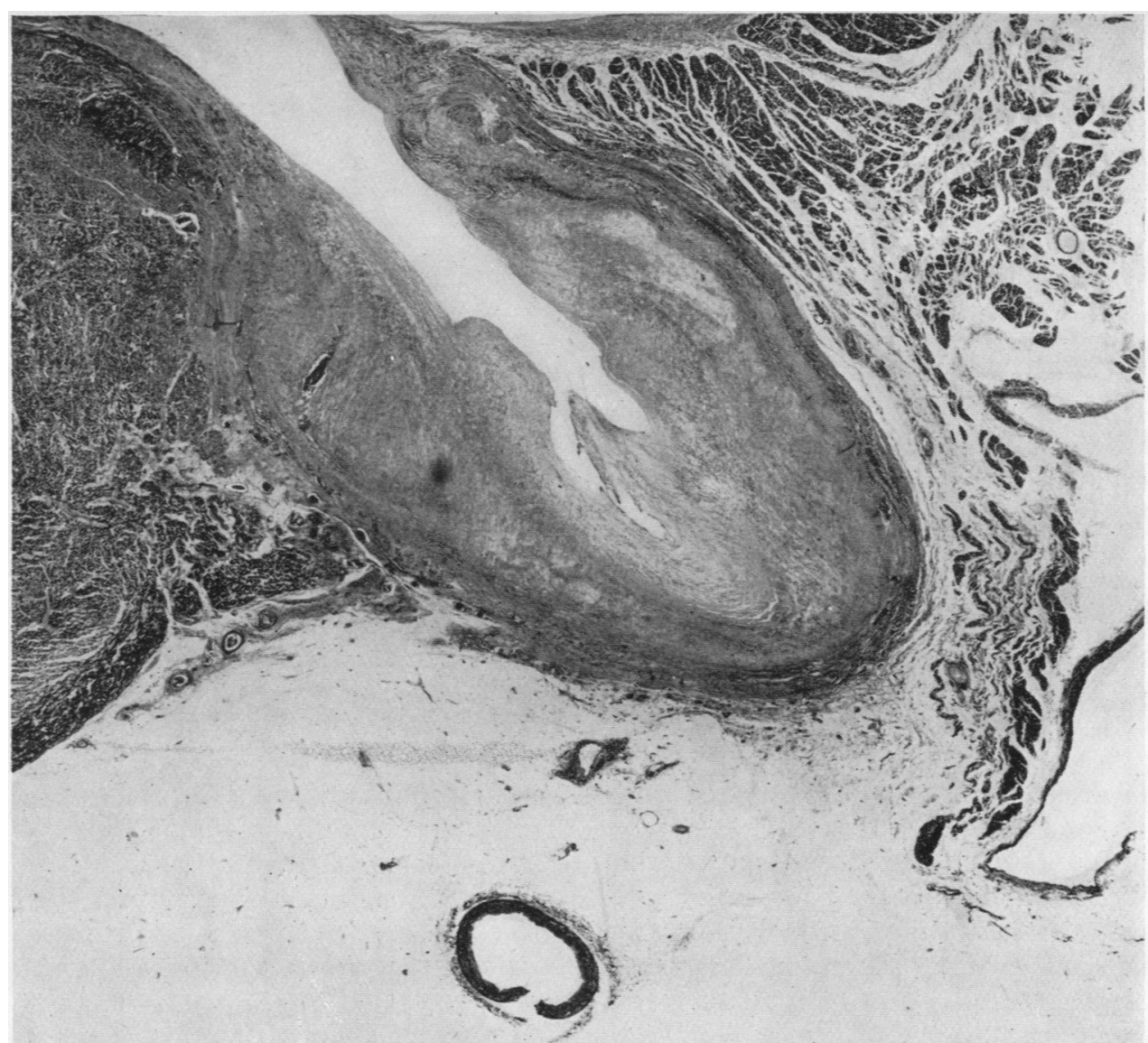

Fig. 4.-This shows the fibrous gutter between the left ventricle and atrium. The origin of the inferior mitral cusp can be observed at the upper border of the photograph. The right coronary artery is noted at the lower portion and the coronary sinus is seen to the right. (Masson $\times$ 7.5.)



FIG. 5.-This section of the myocardium of the left ventricle shows infiltration by lymphocytes, monocytes, and a few eosinophils. In addition, some degenerating muscle cells are noted surrounded by the infiltrate. (Haematoxylin and eosin $\times 485$.) 
this nature (Parkinson, Bedford, and Thomson, 1938 ; and Burn, Hollander, and Crawford, 1943).

Congenital aneurysms are either diverticula of the heart or are related to the interventricular septum (Abbott, 1936). Other aneurysms described have been associated with the sinuses of Valsalva (Micks, 1940). Sometimes aneurysms related to anomalies of the coronary arteries are described as cardiac (Bland, White, and Garland, 1933).

Traumatic aneurysms are not common, but cases are described by Crawford (1943) and Thaon (1947).

In our case there was no history or pathological evidence of coronary thrombosis and infarction, and the important lesion appeared to be obliterative endarteritis in the small arteries and arterioles, only slight intimal thickening being apparent in the larger radicals of the coronary arteries.

Coronary thrombosis is very rare in the South African Bantu. Becker (1946) reported only one case in a pure Bantu in over 3,000 consecutive necropsies on coloured and Bantu subjects.

The lesions and clinical findings did not suggest syphilis and no evidence of bacterial endocarditis or traumatic damage was found.

It is possible that the aneurysm was due to a congenital weakness in the mitral ring, the vascular changes being secondary to pressure from the sac. However, the presence of the lesions at the base of the inferior mitral cusp and the generalized fibrosis and cellular infiltration of the myocardium renders this explanation improbable.

The obliterative endarteritis in the small arteries was not unlike that sometimes noted in chronic rheumatic endocarditis, but none of the other lesions found in this disease were observed, no lesions being present in the valves or endocardium and no Aschoff bodies in many sections. The nature of the myocarditis is uncertain. There was no clinical evidence of diphtheria and the degenerative myocardial changes appeared to be secondary to the cellular infiltration. A diffuse myocarditis is described very rarely in tuberculosis (Saphir, 1941), but in this case the histological changes in no way resembled this disease, and acid-fast bacilli were absent. As no other lesions were observed which afford an adequate explanation for the myocarditis, the case may be described as "isolated " or "Fiedler's" myocarditis. Isolated myocarditis in the South African Bantu does not appear to be uncommon, and we have already seen two other cases in 300 consecutive necropsies; in none was any cause observed.

Although secondary vascular lesions appear very rare in this condition, in this case we considered that they were secondary to the myocardial inflammation, postulating a sequence of events as follows. The obliterative changes in the small blood vessels led to a localized area of ischaemic fibrosis around the inferior part of the mitral ring. The aneurysm was then formed in this area in the same manner as those arising from an area of fibrosis after coronary occlusion.

Summary

A case of non-specific myocarditis and cardiac aneurysm is described in a young adult. The cause of the myocarditis and aneurysm could not be established with certainty. 
A short discussion and review of aneurysms in young adults are given.

Our thanks are due to Dr. Vernon Wilson for permission to publish the clinical findings in this case, to Dr. B. J. P. Becker for invaluable criticism, and to Mr. F A. Brandt for the photographs.

\section{REFERENCES}

Abbott, M. (1927). In Osler's Modern Medicine, 3rd ed., ed. McCrae, T., vol. 4, London. (1936). Atlas of Congenital Cardiac Disease, New York.

Becker, B. J. P. (1946). S. Afr. J. med. Sci., 11, 97.

Benson, R. L., Hunter, W. C., and Manlove, C. H. (1933). Amer. J. Path., 9, 295.

Berlin, R., and Hallen, L. (1938). Acta med. scand., 95, 34.

Bland, E. F., White, P. D., and Garland, J. (1933). Amer. Heart J., 8, 787.

Burn, C. G., Hollander, A. G., and Crawford, J. H. (1943). Ibid., 26, 415.

Crawford, J. H. (1943). Arch. intern. Med., 71, 502.

Dressler, W., and Pfeiffer, R. (1940). Ann. intern. Med., 14, 100.

Macfie, J. W. S., and Ingram, A. (1920). Ann. trop. Med. Parasit., 14, 147.

Micks, R. H. (1940). Brit. Heart J., $2,63$.

Parkinson, J., Bedford, D. E., and Thomson, W. A. R. (1938). Quart. J. Med., 7, 455.

Roessler, W. (1944). Dtsch. Z. Chir., 258, 561.

Sanes, S., and Kenny, F. E. (1934). Amer. J. Dis. Child., 48, 113.

Saphir, O. (1941). Arch. Path., 32, 1000.

Sohval, A. R. (1935). Ibid., $20,429$.

Sternberg, M. (1914). Das chronische partielle Herzaneurysma. Anatomie, Klinik, Diagnosis. Vienna.

Thaon, M. (1947). Arch. Mal. Ca ur., 40, 179.

Vivas-Salas, E. (1948). Amer. J. Dis. Child., 75, 92. 